\title{
Measurement and simulation of anisotropy in the infrared and Raman spectra of $\boldsymbol{\beta}-\mathrm{FeSi}_{2}$ single crystals
}

\author{
G. Guizzetti, F. Marabelli, M. Patrini, and P. Pellegrino \\ Istituto Nazionale per la Fisica della Materia and Dipartimento di Fisica 'A. Volta'” dell' Università degli Studi di Pavia, \\ via Bassi 6, 27100 Pavia, Italy \\ B. Pivac \\ Ruder Boskovic Institute, P.O. Box 1016, Bijenicka c.54, HR-10000 Zagreb, Croatia \\ L. Miglio \\ Istituto Nazionale per la Fisica della Materia and Dipartimento di Fisica dell'Università degli Studi di Milano, \\ via Celoria 16, 20133 Milano, Italy \\ V. Meregalli \\ MPI-FKF, Heisenbergstrasse 1, D-70506 Stuttgart 80, Germany \\ H. Lange and W. Henrion \\ Abteilung Photovoltaik, Hahn-Meitner-Institut Berlin GmbH, Rudower Chaussee 5, D-12489 Berlin, Germany \\ V. Tomm \\ Abteilung Solare Energetik, Hahn-Meitner-Institut Berlin GmbH, Rudower Chaussee 5, D-12489 Berlin, Germany
}

(Received 12 August 1996)

\begin{abstract}
In this paper we show that the orthorhombic phase of $\mathrm{FeSi}_{2}$ (stable at room temperature) displays a sizable anisotropy in the infrared spectra, with minor effects in the Raman data too. This fact is not trivial at all, since the crystal structure corresponds to a moderate distortion of the fluorite symmetry. Our analysis is carried out on small single crystals grown by flux transport, through polarization-resolved far-infrared reflectivity and Raman measurements. Their interpretation has been obtained by means of the simulated spectra with tightbinding molecular dynamics. [S0163-1829(97)05418-0]
\end{abstract}

\section{INTRODUCTION}

$\mathrm{FeSi}_{2}$ is a very interesting material from the fundamental point of view, since it displays several metallic phases in the epitaxial configuration (pseudomorphism), whereas the room-temperature bulk stable structure is surprisingly semiconducting. The latter, orthorhombic $\beta-\mathrm{FeSi}_{2}$, is originated by a moderate distortion of the fluorite metallic phase. ${ }^{1}$ This feature has raised strong technological interest in the last few years, since a direct energy gap as large as $0.85 \mathrm{eV}$ was found at room temperature, matching quite well the transmission window of $\mathrm{SiO}_{2}$ optical fibers at $1.55 \mu \mathrm{m} .^{2}$ The possibility to grow epitaxial $\beta$-FeSi $i_{2}$ films on both (111) and (001) Si substrates discloses interesting perspectives in the fabrication of optoelectronic devices and infrared detectors compatible with silicon technology. ${ }^{3-6}$ Anyway, in spite of such promising features and of the large efforts spent in improving the quality of the films, neither efficient luminescence nor photoconductivity occurs, the lattice matching with the silicon substrate is rather bad, and the carrier mobilities measured on thin films seem to be lower than expected. $^{6}$ Very recently, however, some luminescence has been successfully measured in ion-beam-synthetized samples, ${ }^{7}$ whereas larger mobilities (up to 60 times larger than in films) and low-temperature photoconductivity were observed in single-crystalline specimens. ${ }^{8}$ Furthermore, the- oretical studies emphasized that if an indirect gap is present in the equilibrium configuration, slightly smaller than the direct one, ${ }^{9}$ the situation may be reversed by the presence of strain, due to the large coupling between the electronic bands and the lattice distortions. ${ }^{10}$

Therefore, it is clear that a better understanding of $\beta-\mathrm{FeSi}_{2}$ single crystals is necessary in order to assess the intrinsic properties and to share the new effects induced by the epitaxial configuration. This was a difficult target up to a few years ago, since it was not possible to grow suitable $\beta-\mathrm{FeSi}_{2}$ single crystals by ordinary methods, due to the occurrence of $\mathrm{Si}$ precipitates, originated by the substoichiometry in iron of the high-temperature phase, $\alpha$ - $\mathrm{FeSi}_{2}$, straightforwardly obtained from the melt. Anyway, small single crystals can be synthetized by the flux transport method. Then, since the vibrational spectroscopy is particularly sensitive to stress and anisotropy effects, it is worthwhile to get polarization-resolved spectra from these samples and to compare the results with the epitaxial case. Actually, the dependence of phonon spectra on crystal orientation, thickness, growth, and annealing temperature has already been investigated on $\beta-\mathrm{FeSi}_{2}$ epitaxial films grown by molecular beam epitaxy (MBE) on (111) and (001) Si substrates by using Fourier transform infrared (FTIR) reflectance and transmittance. ${ }^{11}$ Anisotropic effects in Raman measurements on films or polycrystalline samples have been reported too. ${ }^{12}$ 
Therefore, the identification of the typical spectral features in the optical response for each crystallographic direction would allow a better discrimination among the different physical effects affecting the spectra and a more precise morphological and elastic characterization of thin films.

In this paper we report the experimental results of farinfrared (FIR) reflectivity and Raman measurements with polarized light on small single-crystalline specimens obtained by flux growth. The vibrational properties have been investigated in the frequency range $100-700 \mathrm{~cm}^{-1}$, and we found remarkable differences between the IR spectra taken at different polarizations with respect to the crystal axis. Actually, a clear understanding of such complex spectra may arise just on the basis of the comparison to model predictions. Due to the large number of atoms per unit cell, the calculation of the vibrational modes cannot be accomplished by the usual methods of lattice dynamics, since the estimation of the force constant matrix for such a large low-symmetry unit cell becomes prohibitively cumbersome. This is the reason we adopt an alternative strategy in the calculation of the IR and Raman spectra for large structures, i.e., the evaluation of suitable autocorrelation functions during the molecular dynamics evolution of a well-equilibrated sample. The results have also been interpreted on the basis of the experimental spectra for the cubic fluorite structure (typical of $\gamma-\mathrm{FeSi}_{2}$ and of stable $\mathrm{CoSi}_{2}$ and $\mathrm{NiSi}_{2}$ ), taking into account the structural distortion in passing to the $\beta-\mathrm{FeSi}_{2}$ phase.

\section{CRYSTAL STRUCTURE AND SAMPLE PREPARATION}

The conventional orthorhombic cell of $\beta-\mathrm{FeSi}_{2} \quad(a$ $=0.9863 \mathrm{~nm}, b=0.7791 \mathrm{~nm}$, and $c=0.7833 \mathrm{~nm})($ Ref. 13) contains 48 atoms (16 formula units) and is generated by a cooperative Jahn-Teller distortion of the fluorite structure. The latter can be easily figured out by considering the deformation of the tetragonal nucleus of a cubic supercell made up by $8 \mathrm{fcc}$ units, where $a$ is twice the fcc edge along $x$ and $b=c$ are the diagonals of the fcc square faces: $a$ gets shorter, and $b$ and $c$ increase by slightly different amounts. Actually, the space group is $D_{2 h}^{18}$, which corresponds to the primitive cell of 24 atoms with two Bravais sites in the orthorhombic base-centered cell, but a useful comparison to the fluorite structure is obtained by looking at the conventional orthorhombic cell. In Fig. 1 we report a threedimensional (3D) view of it which points out the relevant aspects of the atomic displacements. The variation of first neighbor distances and bond angles is not dramatic: still, relevant changes in the secondary coordination occur, as displayed in Figs. 2 and 3. Flux transport growth generates thin crystalline needles and plaquets: the latter display a definite growth orientation with $b$ and $c$ axes in the basal plane and the $a$ axis perpendicular to it, as evidenced by x-ray diffraction (XRD). The needles, on the contrary, show the $a$ axis perpendicular to the needle axis, but $b$ and $c$ can both occur along this direction. Actually, for the latter case only the $a$ posteriori comparison of the optical spectra to the complementary ones of the plaquets and the simulated spectra can resolve this partial ambiguity.

The crystals were grown in closed ampoules using iodine as the transporting gas. The iodine concentration was $4 \mathrm{mg} \mathrm{I} / \mathrm{cm}^{3}$. The ampoule was placed in a temperature gra- (a)

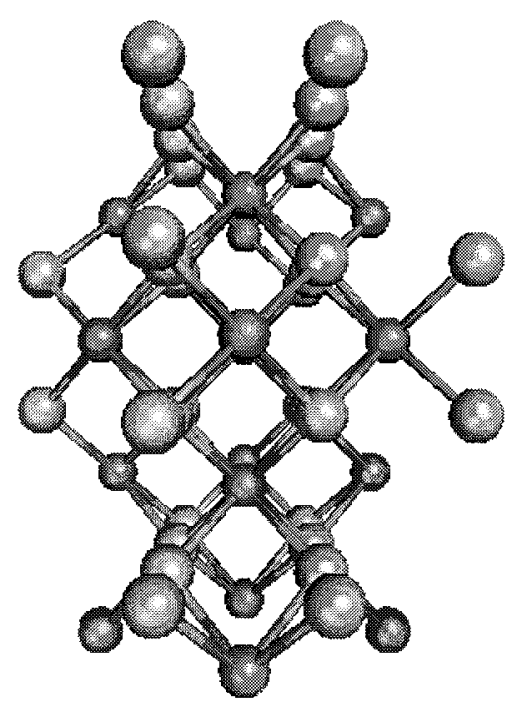

(b)

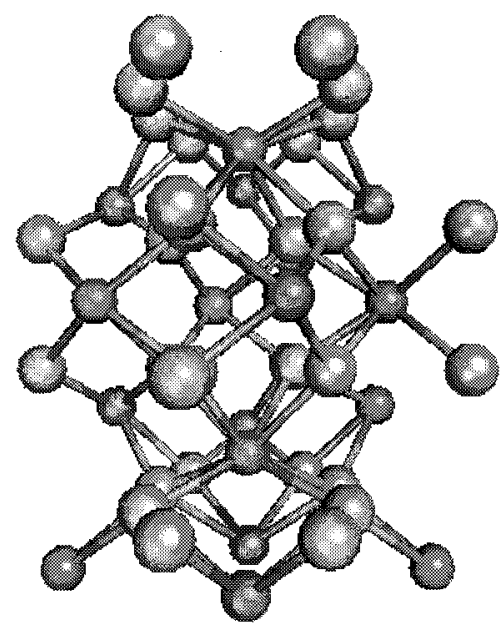

FIG. 1. Perspective view of the orthorhombic cell of $\beta-\mathrm{FeSi}_{2}$ (b) as compared to the original fluorite structure (a). The $x$ direction is topside down, and the darker smaller spheres correspond to the iron atoms.

dient between 950 and $680^{\circ} \mathrm{C}$. Crystals grew at the colder part of the ampoule, at a temperature of about $680^{\circ} \mathrm{C}$, after about $72 \mathrm{~h}$. A single-crystalline plaquet with a natural smooth and shining surface of about $2 \times 3 \mathrm{~mm}^{2}$ (perpendicular to the $a$ axis) and needlelike specimens with an average size of about $0.2 \times 0.2 \times 3 \mathrm{~mm}^{3}$ were used for the optical characterization. A certain amount of impurities, particularly of chromium, is anyway present in the samples at a concentration of $10^{18} \mathrm{~cm}^{-3}$, as determined by electron paramagnetic resonance (EPR). All samples are $p$ type, and their electrical characterization (conductivity and Hall) gives the values of hole concentration $\left(1 \times 10^{18} \mathrm{~cm}^{-3}\right)$ and mobility $\left(20 \mathrm{~cm}^{2} / \mathrm{V} \mathrm{s}\right)$.

\section{OPTICAL CHARACTERIZATION}

\section{A. IR measurements}

Recent band-structure calculations predicted a small anisotropy in the electronic properties between the $a$ axis and the other ones, ${ }^{14}$ but we will see that in the IR spectra a sizable anisotropy between the $b$ and $c$ axes is found too. 

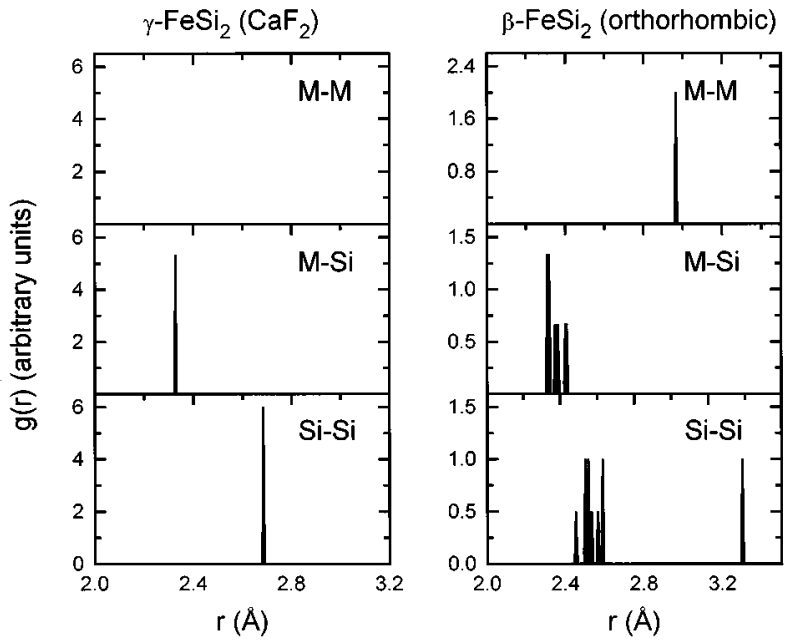

FIG. 2. Pair distribution as a function of the interatomic distance for metal-metal $(M M)$, metal-silicon $(M-\mathrm{Si})$, and silicon-silicon (Si-Si) pairs, both in fluorite and orthorhombic structures.

Far infrared reflectance was measured using a Fourier transform spectrometer (model Bruker IFS 113v). A gold mirror was used as a reference. The measurements on the plaquet were performed with light polarized along the crystallographic $b$ and $c$ axes and gave the spectra shown in Fig. 4(a).

In contrast to what is expected, a clear anisotropy can be noticed. Actually, the crystallographic configuration along the two directions is quite different, as evidenced in Fig. 1 (b). It is then reasonable to find slightly different frequencies for the lattice vibrations along the two directions, due to their higher sensitivity to the structural configuration with respect to the electronic properties.

In order to obtain the complex dielectric function $\varepsilon(\omega)$ $=\varepsilon_{1}(\omega)+i \varepsilon_{2}(\omega)$ and the absorption coefficient, KramersKronig transformation of the reflectivity data has been done. A constant-reflectivity tail at the lowest as well at the highest frequencies was used, and attention was paid that the exten-
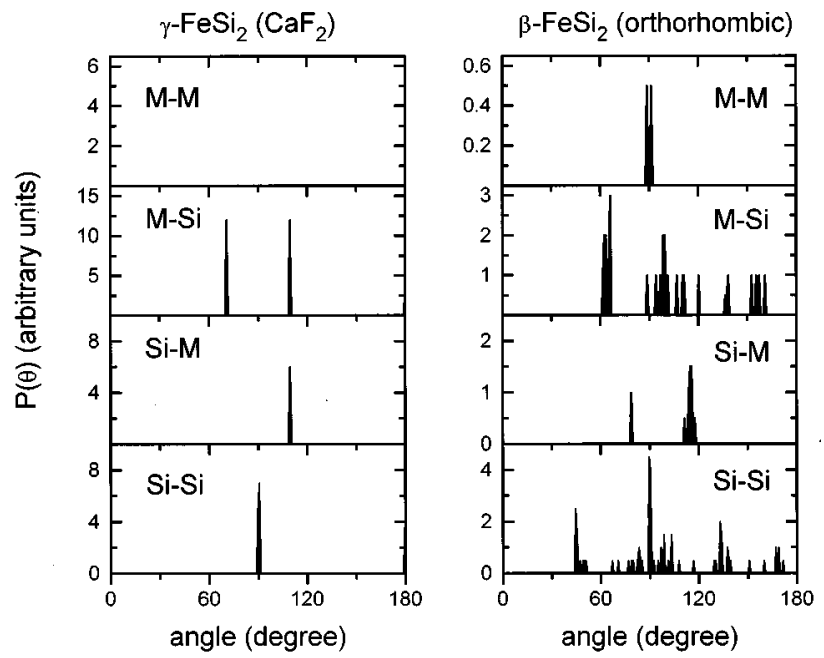

FIG. 3. Same as Fig. 2 for the bond angle distribution as a function of the interbonding angle (the first atomic species indicates the pivot atom).

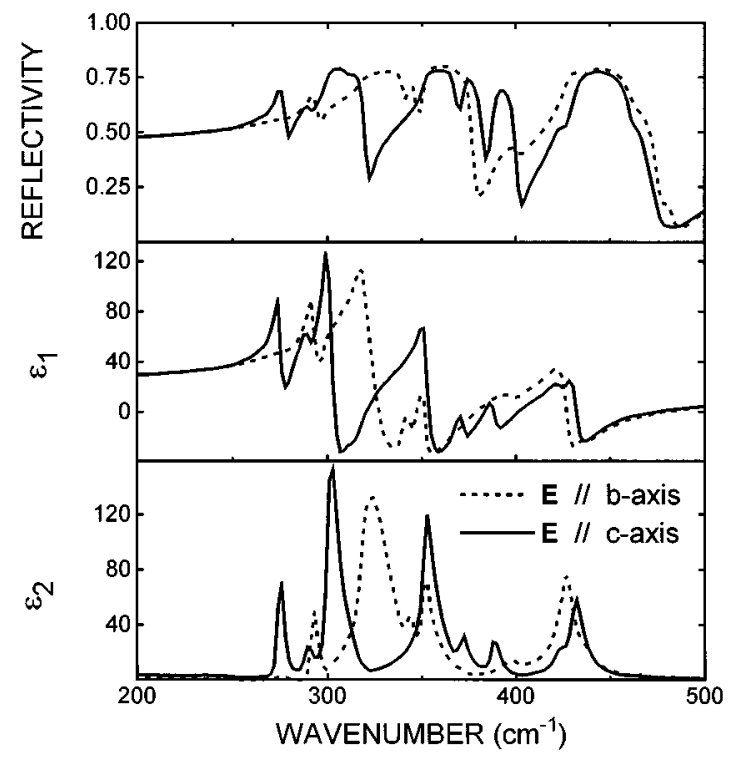

FIG. 4. Reflectivity and real $\left(\varepsilon_{1}\right)$ and imaginary $\left(\varepsilon_{2}\right)$ parts of the dielectric function in the FIR for two different polarizations for a single-crystalline $\beta$-FeSi 2 plaquet.

sion of the tails does not affect the results. Figures 4(b) and 4(c) report the dielectric function so obtained.

A more complex procedure was necessary for the needlelike samples. Since the long $(a)$ crystallographic axis lies perpendicular to the needle length, we investigated several facets on different samples using a spectroscopic ellipsometer in the visible-wavelength range equipped with a micro focusing system. In this way we selected the surfaces which exhibit an anisotropic optical response.

No spectral changes have been observed if one is measuring a single specimen or a collection of samples aligned in the same direction; then, we can reasonably exclude spurious effects related to the small size of samples and assume that their response is quite similar. Therefore, in order to increase the signal intensity, we used for the IR measurements a collection of several samples aligned one beside the other and glued together. The pellet of glue and samples so obtained was then polished with diamond powder in order to obtain a flat surface with the maximum area of exposed samples. The reflectance of such a pellet was measured with polarized light. The contribution of the glue to the signal was observed to be negligible since the glue reflectance was below the detection sensitivity of our instrument. In order to improve the signal-to-noise ratio a large number $(15000)$ of scans was taken during the measurements. The samples were measured with the long axis parallel to the polarization and successively rotated by $90^{\circ}$ in order to measure the response with the axis perpendicular to the polarization. The results obtained for the two orientations are shown in Fig. 5(a).

The reflectance measured for the polarization parallel to the needles resulted in being very similar to the reflectance obtained for a well-oriented sample with the surface perpendicular to the $a$ axis $^{11}$ (see below). Then, the reflectance data so obtained have been scaled on the reflectance of the film, in order to normalize the intensity to the surface area of the sample, and Kramers-Kronig transformed in order to obtain the complex dielectric function [Figs. 5(b) and 5(c)]. 


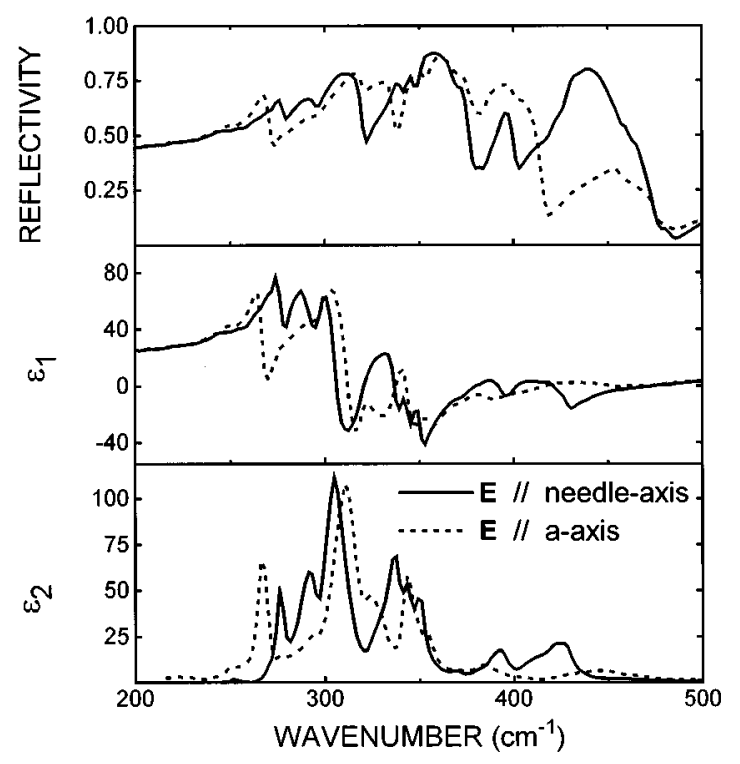

FIG. 5. Reflectivity and real $\left(\varepsilon_{1}\right)$ and imaginary $\left(\varepsilon_{2}\right)$ parts of the dielectric function in the FIR for the two different polarizations parallel and perpendicular to $\beta-\mathrm{FeSi}_{2}$ needle samples.

A clear anisotropy can be noticed in the IR measurements. The scheme of structures in the IR spectra of $\varepsilon_{2}$ is similar for the three different polarizations corresponding to the three crystallographic axes, but the position of the peaks and, in some cases, their relative intensity are different. The first peak at the lowest frequencies is observed at $267 \mathrm{~cm}^{-1}$ for the $a$ direction, at $275 \mathrm{~cm}^{-1}$ for the $c$ axis, and at 293 for the $b$ axis. The main feature observed in all the spectra is also shifted passing from $303 \mathrm{~cm}^{-1}$ ( $c$ axis), to $311 \mathrm{~cm}^{-1}$ (a axis), and $324 \mathrm{~cm}^{-1}$ (b axis). A structure at about $345 \mathrm{~cm}^{-1}$ in the spectrum corresponding to $\mathbf{E} \| a$ is slightly shifted to higher frequencies for $\mathbf{E} \| b$ and $\mathbf{E} \| c$. Finally, the broad and relatively strong structure noticeable at about $430 \mathrm{~cm}^{-1}$ in the $b$ - and $c$-axis spectra is almost suppressed in the spectrum related to the $a$ axis.

\section{B. Raman measurements}

Raman spectra were excited by COHERENT argon-ion laser model Innova 100-15 and recorded by a computerized triple-stage (additive) monochromator DILOR model Z 24. The laser was operating at $514.5 \mathrm{~nm}$ wavelength in a lightstabilizing mode, and the incident power on the sample was kept about $100 \mathrm{~mW}$. The light beam was focused on the sample in a $50-\mu \mathrm{m}$-diam spot. A $90^{\circ}$ scattering geometry was used, with sample surface placed at a near-grazing angle $\left(\approx 75^{\circ}\right)$. Needlelike samples were oriented with the long axis perpendicular to the scattering plane. The incident light was polarized along a direction perpendicular or parallel to the scattering plane. Scattered light was not analyzed using a polarizer and polarization scrambler due to a very low Raman signal intensity. Spectra were recorded using a spectral slit width of $3 \mathrm{~cm}^{-1}$ in the sequential mode with the step size of $1 \mathrm{~cm}^{-1}$. Additional improvement of the signal-to-noise ratio was obtained collecting several spectra over the same interval.

Problems arose in measuring the plaquet because of its very low thickness (a few tens of $\mu \mathrm{m}$ ) and the consequent

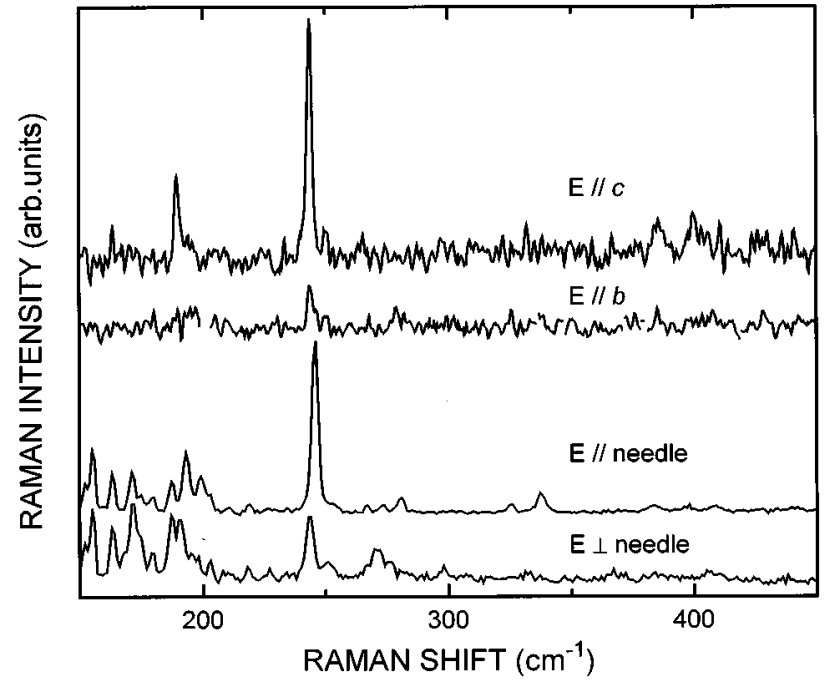

FIG. 6. Raman spectra measured with polarized light on a single-crystalline plaquet and a needle sample of $\beta-\mathrm{FeSi}_{2}$.

thermal instability. Then the corresponding spectra have a poor quality, but anyway sufficient to extract some information.

The spectra obtained for the polarization along the $b$ and $c$ axes (plaquet) or parallel and perpendicular to the needle axis are shown in Fig. 6. The main result concerning $b$ and $c$ directions is the huge enhancement of the main peak at $250 \mathrm{~cm}^{-1}$ when $\mathbf{E} \| c$ with respect to the spectrum corresponding to $\mathbf{E} \| b$. Analogous behavior is shown by the structure at $190 \mathrm{~cm}^{-1}$. A structure typical of the $b$ direction seems to emerge at about $280 \mathrm{~cm}^{-1}$. No large differences can be noticed in the spectra of the needle sample where combinations of $b, c$, and, possibly, $a$ axes have to be taken into account.

At about $270 \mathrm{~cm}^{-1}$ a structure is enhanced when light is polarized perpendicular to the needle with respect to the spectrum corresponding to $\mathbf{E} \|$ needle, whereas the situation is reversed at $340 \mathrm{~cm}^{-1}$. A small, but defined peak at about $300 \mathrm{~cm}^{-1}$ is only present for the polarization perpendicular to the needle (this is the only direction containing the $a$ axis). At the highest frequencies relatively small and broad peaks can be observed in the spectra.

\section{SIMULATIONS}

The basic idea is that each atomic displacement can be represented by the superposition of the normal modes (phonons) in the crystal, so that the spectral features are naturally included in the time evolution of the system. Therefore it is possible to obtain the vibrational density of states and the absorption and Raman scattering spectra by Fourier transforming from time to frequency the autocorrelation functions of the atomic velocity, the derivative of the cell dipole moment and polarizability, respectively:

$$
\begin{gathered}
g(\omega)=A \sum_{i, \alpha} \int_{0}^{\infty}\left\langle m_{i} \nu_{i}^{\alpha}(0) \nu_{i}^{\alpha}(t)\right\rangle \exp (-i \omega t) d t \\
\omega \varepsilon_{2}^{\alpha}(\omega)=B \int_{0}^{\infty}\left\langle\dot{\mu}^{\alpha}(0) \dot{\mu}^{\alpha}(t)\right\rangle \exp (-i \omega t) d t
\end{gathered}
$$




$$
R^{\alpha \beta}(\omega)=B^{\prime} \frac{1}{\hbar \omega} \int_{0}^{\infty}\left\langle\dot{p}^{\alpha \beta}(0) \dot{p}^{\alpha \beta}(t)\right\rangle \exp (-i \omega t) d t,
$$

where $i$ labels the atoms in the cell, $\alpha, \beta$ are the Cartesian components, and $A, B, B^{\prime}$ are suitable constants.

In order to obtain the evolution with time of the system, we integrate the equations of motion for each atom in the simulation cell, and in turn we need the interatomic forces from a suitable potential. The latter is obtained by a semiempirical scheme which we have successfully used for the interpretation of the stability hierarchy in $\mathrm{FeSi}_{2},{ }^{1} \mathrm{FeSi}$, and $\mathrm{CoSi}$ (Ref. 15) epitaxial phases. It is grounded on the partition of the total energy into an attractive part $E_{\mathrm{bs}}$, originated by a summation over occupied tight-binding (TB) states $\varepsilon_{n, k}$ ( $n$ is the band index and $k$ is the wave vector), and a repulsive contribution $E_{\text {rep }}$, which is generated by the summation of one short-range, two-body potential $\phi\left(r_{i j}\right)$ on the relevant shell of neighbors $(j)$ :

$$
E=E_{\mathrm{bs}}+E_{\mathrm{rep}}=\sum_{n k} \varepsilon_{n k}+\sum_{i<j} \phi\left(r_{i j}\right) .
$$

$E_{\mathrm{bs}}$ is negative and takes into account the electronic features, as provided by a Slater-Koster (SK) parametrization of the TB matrix ${ }^{16}$ involving $s$ and $p$ orbitals for silicon and $s$, $p$, and $d$ ones for iron. In the case of tight-binding molecular dynamics the diagonal (onsite) elements are kept frozen, but the hopping elements between neighboring atoms change according to the director cosines and the modulus of $r_{i j} .{ }^{16} \mathrm{In}$ particular, the radial part of the SK parameters scales with the interatomic distance $r_{i j}$ following the Harrison rule ${ }^{17}$

$$
H_{l, l^{\prime}, m}\left(r_{i j}\right)=H_{l, l^{\prime}, m}\left(r_{i j}^{0}\right)\left(r_{i j}^{0} / r_{i j}\right) n_{l, l^{\prime}},
$$

where $l, l^{\prime}=s, p, d ; m=\sigma, \pi, \delta$, and $n_{l, l^{\prime}}=2,3.5$, or 5 if none, one, or both orbitals are $d$-like, respectively. At variance with respect to previous total energy calculations, ${ }^{1,10,15}$ in the case of molecular-dynamics simulations it is compulsory to operate a smooth cutoff of the interactions within the global cutoff radius imposed by the size of the simulation cell. Therefore we multiplied both $H_{l, l^{\prime}, m}(r)$ and the repulsive interactions $\phi(r)$ by

$$
\frac{1}{2}\left[1-\sin \left(\frac{\pi}{2} \frac{r-r_{c}}{w}\right)\right],
$$

where $r_{c}+w$ is the cutoff radius. In fact, below $r=r_{c}-w$ the cutoff function is set to 1 and above $r=r_{c}+w$ it is 0 .

Here we just mention that small differences in the equilibrium positions of the atoms in the (fixed) orthorhombic cell may arise depending on the cutoff radius for the interactions, which is $3.2-3.3 \AA$ in our case. This, in turn, may change the degeneracy of some peaks, as due to a symmetry lowering mostly affecting the secondary coordination of silicon sites.

The repulsive term phenomenologically represents the quantum-mechanical interaction between occupied orbitals, and the summation of $\phi\left(r_{i j}\right)$ is extended as for the TB elements. We have chosen a simple two-body form with a power-law dependence on the interatomic distance:

$$
\phi\left(r_{i j}\right)=\phi_{i j}\left(r_{i j}\right)^{-m},
$$

which, however, discriminates the metallic pairs from the silicon ones with different $\phi_{i j}$ and $m$, whereas a suitable average is taken in the Si-Fe case. ${ }^{10}$ The relevant set of parameters has been already published in Ref. 10, but for the absence of the cutoff function, where total energy calculations for $\mathrm{FeSi}_{2}$ are reported.

The forces entering the molecular-dynamics simulation are calculated by a straightforward derivative of the repulsive potential and a Hellman-Feynman estimation of the attractive part, retaining only $k=0$ terms, ${ }^{18}$

$$
F_{i, \alpha}^{b s}=-\sum_{n, k} f_{n, k} \frac{\partial}{\partial r_{i, \alpha}}\langle n, k|H| n, k\rangle,
$$

where $i$ and $\alpha$ label the atom and Cartesian component, respectively, $f$ is the Fermi function, and $H$ is the total TB element, cutoff function included.

The simulations have been performed for a nonprimitive orthorhombic cell containing 48 atoms, with periodic boundary conditions. Actually, the primitive choice would correspond to 24 atoms in a one-face-centered orthorhombic cell, but the necessity to have a cutoff sphere totally included in the simulation cell prevented us from reducing the cell size. So our vibrational spectra include folded modes not pertaining to the primitive cell, but the IR and Raman spectra should correspond to the experimental ones at the $\Gamma$ point for symmetry reasons, as far as the equilibrium positions in the simulated sample actually give rise to a $D_{2 h}^{18}$ space group.

Time evolution of the system is obtained by integrating the Newton equations with a velocity-Verlet algorithm and a time step $\tau$ as large as $1 \mathrm{fs}$, which allows for an energy conservation during constant energy (nve) microcanonic runs as good as $1 / 10000$. We have performed two simulations with slightly different cutoff parameters $\left(r_{c}=3.1 \AA, w\right.$ $=0.1 \AA$ and $r_{c}=3.15 \AA, w=0.15 \AA$ ) by taking the experimental structure, heating it up to $100 \mathrm{~K}$ during a constant temperature $(n v t)$ canonic run of $1000 \tau$ where the velocities have been continuously rescaled. Then we performed an $n v e$ run as long as $30000 \tau$, where the collection of data (positions and velocities in the cell) has been possible as the sample resulted in being well equilibrated. Actually, we stored $\nu_{i}^{\alpha}, \dot{\mu}^{\alpha}, \dot{p}^{\alpha \beta}$ every $10 \tau$, which means that our sampling of the lowest frequency $\left(250 \mathrm{~cm}^{-1}\right)$ was 13.4 times per period for 224 oscillations and 7.4 times per period for 405 oscillations in the case of the highest frequency $\left(450 \mathrm{~cm}^{-1}\right)$. The derivative of the dipole moment in the simulation cell is obtained by weighting all the atomic velocities with the static charges for the equilibrium configuration $(\mathrm{Fe} \cong-2 e, \mathrm{Si} \cong e$, probably an overestimation due to the tight-binding scheme). The derivative of the cell polarizability, on the other hand, is calculated in the framework of the bond polarizability model, where only first-neighbor bonds between $\mathrm{Fe}$ and $\mathrm{Si}$ are considered. The latter contribute to the total polarizability by a parallel component $\alpha_{\|}$and a perpendicular one $\alpha_{\perp}$, which depend on the bond length. Symmetric and antisymmetric contributions can be constructed in order to simplify the final expression of the Raman tensor and the identification of the leading term

$$
\begin{aligned}
3 \alpha_{s} & =\left(\alpha_{\|}+2 \alpha_{\perp}\right), \\
\alpha_{a} & =\left(\alpha_{\|}-\alpha_{\perp}\right) .
\end{aligned}
$$


The time derivative of the cell polarizability is obtained by deriving a sum of bond polarizabilities, which are expanded in terms of atomic displacements: $\quad p_{\alpha \beta}^{\prime}$ is finally obtained as the sum of three terms, proportional to $d \alpha_{s} / d r, d \alpha_{a} / d r$, and $\alpha_{a}$, respectively. ${ }^{19}$ No one of the latter is known, even qualitatively; still, by estimating the Raman spectra at any incoming-outgoing polarization for each term separately, we found that the first one gives rise to no anisotropy at all and that only the third term gives Raman spectra which are in agreement with the experimental findings. Therefore we concluded that $d \alpha_{s} / d r$ and $d \alpha_{a} / d r$ are negligibly small.

The identification of the character pertaining to the different features has been done by performing the same simulations described above with iron and silicon masses multiplied by 100 , alternatively. In this way a simulated isotopic shift is produced and we have been able to share which peaks involve iron movement only, which ones involve just silicon displacements, and which ones are produced by both. Obviously, this is not a real symmetry analysis of the character, but still it helps in the interpretation of the spectra and points out the flexibility of the simulation method in addressing complex structures.

It is understood that our results can be taken as a qualitative indication of the occurring trends from one polarization to the other, since several approximations in the model may affect both the intensity and position of the peaks. For what concerns the dynamics, the scaling laws with interatomic distance and the central character of the repulsive potential are not checked to elastic or optical data, and only the a posteriori agreement to the experimental frequencies (as in our case) may indicate that a fitting on lattice parameters and bulk modula is really sufficient. Moreover, the IR and Raman spectra are mediated by dielectric models, which may contain few approximations and several parameters, as for the case of the bond polarizability model. This is true even in the case of the IR spectra, where the effective charges entering the dipole moments are not consistently calculated at each time step. They are fixed and taken as the total charge provided by the tight-binding occupation number at equilibrium. Still, this is the only way to avoid misdefinition of the cell polarization due to charge flow across the cell boundary (otherwise a Barry phase approach is necessary). Actually, we did not include the Coulombic restoring forces provided by longitudinal charge displacements to our dynamics, still, we think that it does not affect either IR or Raman spectra. Finally, the limited range of the interaction (within the cutoff radius) leaves out part of $\mathrm{Fe}-\mathrm{Fe}$ third-neighbor couplings, so that an underestimation of the modes involving counterphase motion of them is very likely.

\section{RESULTS AND DISCUSSION}

As we see from Figs. 1-3, the structural deformation from fluorite to the $\beta$ phase is characterized by a distortion of the cubic cage of first-neighbor silicon atoms around the metal sites and by a sizable change in the coordination of second and third neighbors. This explains the more than eightfold increase in volume of the primitive cell and the corresponding folding of the Brillouin zone. Therefore the interpretation of both Infrared and Raman modes can be roughly done in terms of the spectral features in the fluorite

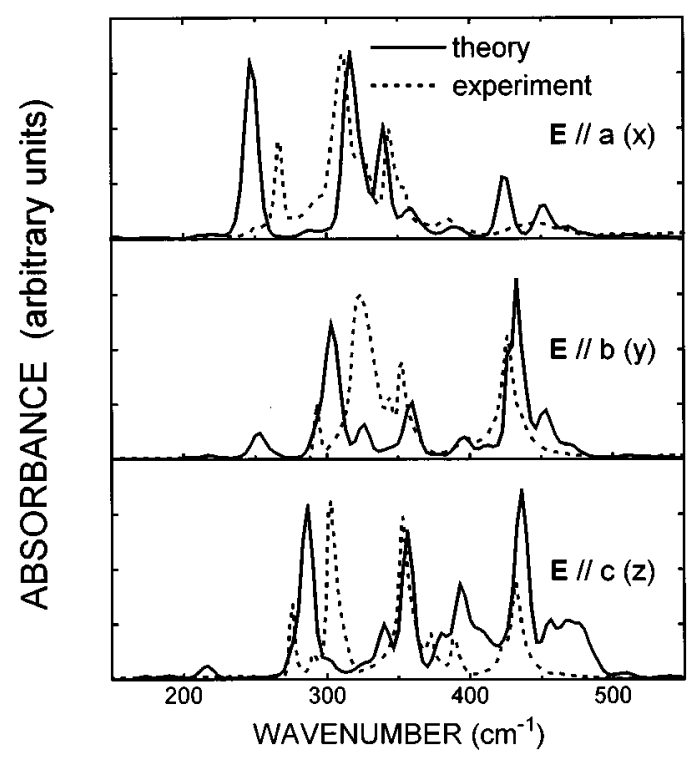

FIG. 7. Calculated (solid line) and experimental (dotted line) absorption coefficient for the $x(\mathrm{a}), y(\mathrm{~b})$, and $z$ (c) polarizations.

structure, both at the zone center (intra-formula-unit modes) and outside it (folded modes or the inter-formula-unit modes). Actually, there are no experimental data for fluorite $\mathrm{FeSi}_{2}$, since it is not bulk stable and the epitaxial films are too thin. However, comparison of the existing experimental and theoretical data for fluorite $\mathrm{CoSi}_{2}$ is very helpful ${ }^{20-22}$ as the frequencies for ideal (epitaxially stabilized) $\mathrm{FeSi}_{2}$ should be very similar. So we expect the main IR modes involving counterphase motion of $\mathrm{Fe}$ and $\mathrm{Si}$ atoms to be positioned around $300-350 \mathrm{~cm}^{-1}$, probably as a multiplet originated by the zone folding from fluorite to orthorhombic. The Raman mode originated by the fluorite structure (only Fe moving) should be found around $250-270 \mathrm{~cm}^{-1}$. These peaks are clearly visible and outstanding in the IR spectra [Figs. 4(c) and 5(c)] and the Raman spectra (Fig. 6), independently of any anisotropy effect.

In Fig. 7 we show the experimental absorption coefficient for the electric field polarized along $x, y$, and $z$ directions (dotted lines), as compared to the simulated spectra. We note that the agreement is rather good, especially between 350 and $400 \mathrm{~cm}^{-1}$, and that the trends in peak intensity and position in passing from one polarization to the other are nicely reproduced. In fact, we confirm that the spectra along $x, y$, and $z$ directions are pretty different, even for what concerns the two latter cases, which were supposed to be very similar. By considering the $x$ direction more in detail, we note that the main doublet inbetween 300 and $350 \mathrm{~cm}^{-1}$ and the small structure just below $400 \mathrm{~cm}^{-1}$ originated from $\mathrm{Fe}$ and $\mathrm{Si} \mathrm{mo-}$ tion, essentially the zone center and the two folded modes (at higher and lower frequencies) of the higher optical branch in fluorite. Beyond $400 \mathrm{~cm}^{-1}$, outside the frequency range for the fluorite structure, we have the experimental broad peak, which corresponds to a doublet in the simulated spectra, probably as an artifact of the double simulation cell, which does not display a perfect $D_{2 h}^{18}$ symmetry. It turns out to originate from a displacement pattern involving $\mathrm{Si}$ atoms only, presumably related to the peculiar distorted structure occurring in the $\beta$ phase. At very low frequencies we find a 


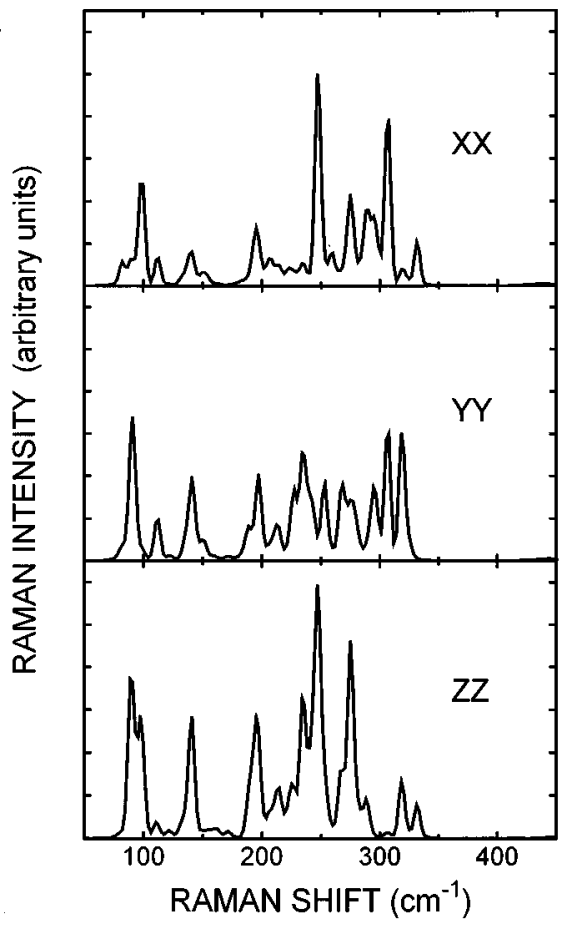

FIG. 8. Calculated Raman spectra for the $x x$ (a), $y y$ (b), and $z z$ (c) components of $\beta-\mathrm{FeSi}_{2}$.

sharp experimental peak at about $260-270 \mathrm{~cm}^{-1}$. It is sizably underestimated in the simulated spectra probably because it involves counterphase motion of Fe atoms only [IR activation of a folded lower (Raman) branch in fluorite], which means that part of the restoring forces involving thirdneighboring $\mathrm{Fe}-\mathrm{Fe}$ is absent, falling outside our cutoff radius.

By moving to the other two polarizations, the interpretation of the spectral features and the quality of the agreement between experimental and simulated spectra still remain, but the peaks are shifted and change intensity, even in passing from $y$ to $z$. Therefore the anisotropy is also present between $b$ and $c$, the two short sides of the orthorhombic cell, which are very similar in size. In fact, from Fig. 1 we note that the arrangement of the atomic positions along these two directions is not the same.

In Fig. 8 we report our calculated Raman components $x x, y y$, and $z z$. Here comparison to the experimental spectra is less meaningful due to the approximations in the polarizability tensor affecting the relative intensities from one side and the lack of polarization for the scattered light from the other. (A further complication could be ascribed to the grazing-angle geometry used in the measurements, which introduces in the polarization of incident light a component perpendicular to the surface.)

Still, we note that both theory and experiments indicate a sizable lowering of the main Raman feature at about $250 \mathrm{~cm}^{-1}$ in passing from $z z$ to $y y$ : a further indication that these two directions are far from being isotropic. In addition to the experimental information, the simulated spectra show that the $x x$ polarization should display a large predominance of the main Raman feature on the other structures. As mentioned before, the structure at about $300 \mathrm{~cm}^{-1}$, occurring in a spectrum taken on the needle, could be related to the $a$ axis and finds a correspondence in the calculated $x x$ spec-

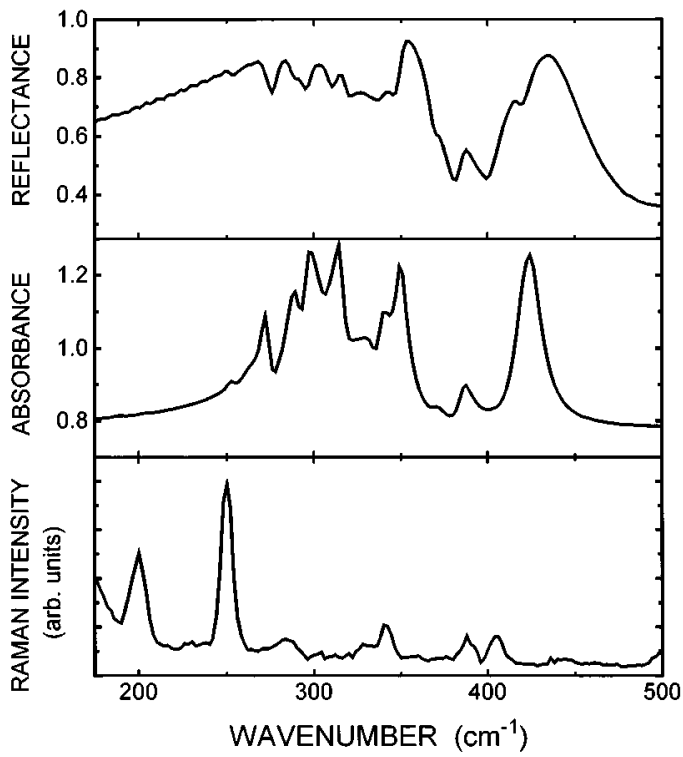

FIG. 9. Reflectance, absorbance, and Raman spectra for the 7500-^̊-thick $\beta$ - $\mathrm{FeSi}_{2}$ film.

trum. This assignment seems to be confirmed by Raman measurements on oriented epitaxial films. For what concerns the highest frequencies, it is worth noting that the simulated spectra do not show any feature beyond $350 \mathrm{~cm}^{-1}$; therefore, the experimental peaks weakly occurring at about $400 \mathrm{~cm}^{-1}$ are more likely to originate from two-phonon processes involving the multiplet at about $200 \mathrm{~cm}^{-1}$ (a relatively strong feature both in simulated and experimental $z z$ spectra).

In Fig. 9 we report the reflectance, absorbance, and Raman spectra of a 7500- $\AA$ FeSi ${ }_{2}$ film obtained by molecular beam epitaxy deposition on $\mathrm{Si}(001)$, as taken from Ref. 11 . The sample was characterized in situ by transmission electron microscopy (TEM) and electron diffraction. It grew with the (100) plane parallel to the silicon surface, i.e., with the long $a$ axis perpendicular to the surface. Therefore, due to the spread of domain orientation in the film and the nonpolarized light used in the experiment, the film absorbance should display both contributions from $y$ and $z$. Actually, by comparison to the polarization, a small contribution of the $x$ component can be identified. In particular, the peak at $314 \mathrm{~cm}^{-1}$ can be understood as a superposition of $y$ and $x$ contributions and the small shoulder at $260 \mathrm{~cm}^{-1}$ can originate from the $x$ component. The other structures can be easily connected, apart from slight shifts, with the observed ones in $\mathbf{E} \| b$ and $\mathbf{E} \| c$ spectra. Therefore the IR analysis is able to characterize the epitaxial sample and to point out the probably minority existence of misoriented grains.

The Raman spectrum exhibits a worse resolution with respect to the single-crystal ones and a slight shift of the structures to higher frequencies (it could also be temperature induced). Here it is not possible to share the contributions from $x$ and $z$ polarization: still, it is clear from the intensity of the peak at $250 \mathrm{~cm}^{-1}$ and the one at $200 \mathrm{~cm}^{-1}$ that one or both of them should be present at a relevant percentage.

In conclusion, anisotropy effects can be clearly observed in the vibrational spectra of $\beta-\mathrm{FeSi}_{2}$ to a much larger extent than the electronic spectra, as due to the enhanced sensitivity 
of the infrared and Raman features to the local structural distortions. The resolution in peaks shifts and the accuracy in their intensity allow an efficient characterization of the epitaxial samples and disclose the possibility of a more accurate analysis of strain effects depending on the growth conditions.

\section{ACKNOWLEDGMENTS}

This work has been partially supported by H.C.M. Project No. CHRX-CT930318 of the European Commission and by Volkswagen-Stiftung I 70408.
${ }^{1}$ L. Miglio, F. Tavazza, and G. Malegori, Appl. Phys. Lett. 67, 1 (1995), and references therein.

${ }^{2}$ M. C. Bost and J. E. Mahan, J. Appl. Phys. 58, 2696 (1995); 64, 2034 (1988).

${ }^{3}$ J. Derrien, J. Chevrier, V. Le Thanh, T. E. Crumbaker, J. Y. Natoli, and I. Berbezier, Appl. Surf. Sci. 70-71, 546 (1993).

${ }^{4}$ K. Radermacher, R. Carius, and S. Mantl, Nucl. Instrum. Methods Phys. Res. B 84, 163 (1994).

${ }^{5}$ H. Lange, in Silicide Thin Films-Fabrication, Properties and Applications, edited by R. T. Tung, K. Maex, P. W. Pellegrini, and L. H. Allen, MRS Symposia Proceedings No. 402 (Materials Research Society, Pittsburgh, 1996).

${ }^{6}$ H. von Känel, U. Kafader, P. Sutter, N. Onda, H. Sirringhaus, E. Müller, U. Kroll, C. Schwarz, and S. Goncalves-Conto, in Silicides, Germanides, and Their Interfaces, edited by R. W. Fathauer, S. Mantl, L. J. Schowalter, and K. N. Tu, MRS Symposia Proceedings No. 320 (Materials Research Society, Pittsburgh, 1994), p. 73; D. J. Oostra, C. W. T. Bulle-Lieuwma, D. E. W. Vandenhoudt, F. Felten, and J. C. Jans, J. Appl. Phys. 74, 4347 (1993); N. Kobayashi, H. Katsumata, H. L. Shen, M. Hasegawa, Y. Makita, H. Shibata, S. Kimura, A. Obara, S. Uekusa, and T. Hatano, Thin Solid Films 270, 406 (1995).

${ }^{7}$ D. N. Leong and M. A. Harry, Appl. Phys. Lett. 68, 1649 (1996).

${ }^{8}$ E. Arushanov, Ch. Kloc, and J. E. Bucher, Phys. Rev. B 50, 2653 (1994); E. Arushanov, Ch. Kloc, H. Hohl, and E. Bucher, J. Appl. Phys. 75, 5106 (1994); E. Arushanov, E. Bucher, Ch. Kloc, O. Kulikova, L. Kulyuk, and A. Siminel, Phys. Rev. B 52, 20 (1995).

${ }^{9}$ N. E. Christensen, Phys. Rev. B 42, 7148 (1990); S. Eisebitt, J. E. Rubensson, M. Nicodemus, T. Böske, S. Blügel, W. Eberhardt,
K. Radermacher, S. Mantl, and G. Bihlmeyer, ibid. 50, 18330 (1994).

${ }^{10}$ L. Miglio and G. Malegori, Phys. Rev. B 52, 1448 (1995).

${ }^{11}$ G. Guizzetti, F. Marabelli, M. Patrini, Y. Mo, N. Onda, and H. von Kaenel, in Silicides, Germanides, and Their Interfaces (Ref. 6), pp. 127-132.

${ }^{12}$ K. Lefki, P. Muret, E. Bustarret, N. Boutarek, R. Madar, J. Chevrier, J. Derrien, and M. Brunel, Solid State Commun. 80, 791 (1991).

${ }^{13}$ Y. Dusausoy, J. Protas, R. Wandji, and B. Roques, Acta Crystallogr. Sec. B 27, 1209 (1971).

${ }^{14} \mathrm{~V}$. Antonov (private communication); V. Antonov et al. (unpublished).

${ }^{15}$ H. von Kaenel, C. Schwarz, S. Goncalves-Conto, E. Mueller, L. Miglio, F. Tavazza, and G. Malegori, Phys. Rev. Lett. 74, 1163 (1995).

${ }^{16}$ J. C. Slater and G. F. Koster, Phys. Rev. 94, 1498 (1954).

${ }^{17}$ W. A. Harrison, Electronic Structure and the Properties of Solids (Freeman, San Francisco, 1980).

${ }^{18}$ C. Molteni, L. Colombo, and L. Miglio, Europhys. Lett. 24, 659 (1993); Phys. Rev. B 50, 4371 (1994); J. Phys. Condens. Matter 6, 5243 (1994); 6, 5255 (1994).

${ }^{19}$ R. J. Bell, in Methods in Computational Physics, edited by G. Gilat (Academic, New York, 1976), Vol. 15, p. 215; Leo Miglio (unpublished).

${ }^{20}$ S. Bocelli, G. Guizzetti, F. Marabelli, G. Thungström, and C. S. Petersson, Appl. Surf. Sci. 91, 30 (1995).

${ }^{21}$ S. Sanguinetti, C. Calegari, and L. Miglio, Appl. Surf. Sci. 91, 103 (1995); S. Sanguinetti, C. Calegari, F. Tavazza, G. Benedek, V. Velasco, and Leo Miglio, Phys. Rev. B 54, 9196 (1996).

${ }^{22}$ V. Milman, M. H. Lee, and M. C. Payne, Phys. Rev. B 48, 9223 (1993). 